\title{
Correction to: Absence of EIF1AX, PPM1D, and CHEK2 mutations reported in Thyroid Cancer Genome Atlas (TCGA) in a large series of thyroid cancer
}

\author{
Ali S. Alzahrani ${ }^{1,2} \cdot$ Avaniyapuram Kannan Murugan $^{1} \cdot$ Ebtesam Qasem ${ }^{1} \cdot$ Meshael M. Alswailem ${ }^{1} \cdot$ \\ Balgees AlGhamdi ${ }^{3}$ Yosra Moria ${ }^{2} \cdot$ Hindi Al-Hindi ${ }^{4}$
}

Published online: 25 June 2019

(c) Springer Science+Business Media, LLC, part of Springer Nature 2019

\section{Correction to: Endocrine}

https://doi.org/10.1007/s12020-018-1762-6

Published online 29 September 2018

The original version of this article unfortunately contained a mistake in the abstract and body of the article. The acronym
TCGA should refer to "The Cancer Genome Atlas" not "Thyroid Cancer Genome Atlas". This has been corrected with this erratum.

The original article can be found online at https://doi.org/10.1007/ s12020-018-1762-6

Ali S. Alzahrani

aliz@kfshrc.edu.sa

1 Department of Molecular Oncology, King Faisal Specialist Hospital \& Research Centre, Riyadh, Saudi Arabia

2 Department of Medicine, King Faisal Specialist Hospital \& Research Centre, Riyadh, Saudi Arabia

3 Research Centre-Jeddah, King Faisal Specialist Hospital \& Research Centre, Jeddah, Saudi Arabia

4 Department of Laboratory Medicine and Pathology, King Faisal Specialist Hospital \& Research Centre, Riyadh, Saudi Arabia 